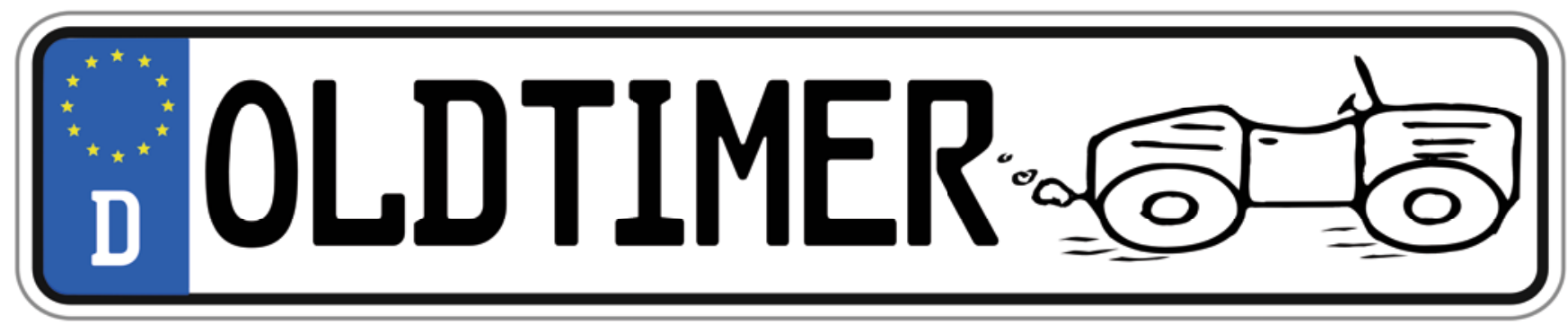

\title{
Wie die - lange Zeit sakrosankte - PsychPV zur Fortschrittsbremse wurde
}

Als Anfang der 1990er-Jahre die Psychiatrie-Personalverordnung (PsychPV) als Finanzierungssystem für stationäre und teilstationäre psychiatrische Leistungen eingeführt wurde, war es ein sensationeller Fortschritt in Richtung medizinisch und menschlich akzeptable Patientenversorgung. Leider wurde damals bei der Konzeption versäumt, finanzielle Veränderungsmöglichkeiten bei evidenzbasierten Fortschritten in der Behandlung psychischer Erkrankungen aufzunehmen. Das heißt, die PsychPV ist seit nahezu 25 Jahren unverändert geblieben und hat personelle Konsequenzen von innovativen leitliniengerechten Behandlungsmethoden nicht vollzogen.

Die in den 1980er-Jahren konzipierte PsychPV ist vornehmlich auf eine psychopharmakologische und sozialpsychiatrische Therapie psychischer Erkrankungen ausgerichtet. Für die Einbeziehung psychotherapeutischer Verfahren in die Krankenhausbehandlung gab es damals nur sehr begrenzte wissenschaftliche Evidenzen. Dies hat sich jedoch insbesondere in den letzten zehn Jahren einschneidend verändert. Inzwischen ist in nahezu allen S3-Leitlinien der wichtigsten psychischen Erkrankungen wie Depressionen, Zwangserkrankungen, Borderlinestörungen, Essstörungen, Angsterkrankungen, Alkoholismus oder schizophrene Erkrankungen die Psychotherapie entweder alleinige sogenannte Firstline-Therapie oder in Kombination mit pharmakologischen und sozialpsychiatrischen Interventionen. Dies kann aber auf dem Boden der PsychPV-Finanzierung bisher nicht umgesetzt werden, da nur etwa eine Stunde Psychotherapie pro Woche pro Patient finanziert wird. Metaanalysen ergeben jedoch, dass in der Regel 20 bis 25 Therapiestunden für eine adäquate Besserung bei den genannten Störungsbildern notwendig ist. Das bedeutet, entweder erfolgt nur ein unzureichendes „Antherapieren“ oder die stationären Behandlungsdauern sind sehr lang. Nur in Universitätskliniken stehen durch Forschungsgelder, Drittmittel und die gar nicht oder nur gering entlohnten psychologischen Psychotherapeuten in Ausbildung als zusätzliches Personal zur Verfügung, um zumindest bei einigen Krankheitsbildern leitliniengerechte Behandlungen durchzuführen.

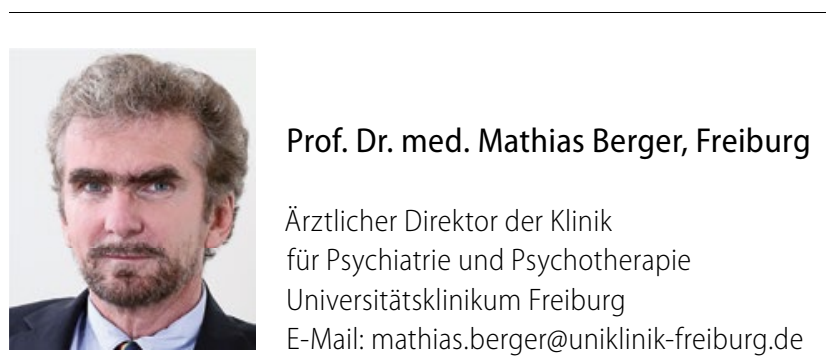

Mit dem jetzt geplanten „Pauschalierten Entgeltsystem in Psychiatrie und Psychosomatik“ (PEPP) ergibt sich beim gegenwärtigen Planungsstand eine noch weitere finanzielle Absenkung für stationäre und teilstationäre Psychotherapie. Dies droht angesichts der unzulänglichen ambulanten psychiatrischen und psychotherapeutischen Versorgungssituation (dargestellt im Editorial der InFo Neurologie \& Psychiatrie, Ausgabe 11/2014).

Im DRG-System, das ja Pate für das PEPP-System sein soll, ist - soweit bekannt - nirgends festgesetzt, dass durch eine Unterfinanzierung Patienten deutlich unterhalb des Niveaus der vorliegenden Leitlinien behandelt werden müssen. Aus medizinischer Sicht ist das PEPP-System in der gegenwärtigen Form strikt abzulehnen. Der jahrzehntelange Kampf um eine wenigstens $100 \%$-PsychPV-Finanzierung der Krankenhäuser, die in der Regel bei $90 \%$ und in vielen Krankenhäusern sogar noch darunter liegt, hat den Blick verschleiert, dass inzwischen auch eine $100 \%$ ige PsychPV-Finanzierung mit einer State-of-the-Art psychiatrisch-psychotherapeutischen Behandlung nicht mehr vereinbar ist. So ist erfreulich, dass sich ein breiter medizinisch begründeter Widerstand gegen eine vor allen Dingen bürokratisch und finanztechnisch begründete Neugestaltung der Finanzierung psychiatrisch-psychosomatisch-psychotherapeutischer Krankenhausleistungen formiert.

Mathias Berger, Freiburg 